\title{
Wrist Rehabilitation Robot System and Its Effectiveness for Patients
}

\author{
Ikuo Yamamoto, ${ }^{1 *}$ Miki Matsui, ${ }^{1}$ Toshio Higashi, ${ }^{1}$ \\ Naoki Iso, ${ }^{1}$ Kenji Hachisuka, ${ }^{2}$ and Akiko Hachisuka ${ }^{3}$ \\ ${ }^{1}$ Nagasaki University, 1-14 Bunkyomachi, Nagasaki 852-8521, Japan \\ ${ }^{2}$ Moji Medical Center, 3-1 Higashiminatomachi, Moji-ku, Kitakyushu-shi, Fukuoka 801-8502, Japan \\ ${ }^{3}$ University of Occupational and Environmental Health, \\ 1-1 Iseigaoka, Yahatanishi-ku Kitakyushu-shi, Fukuoka 807-0804, Japan
}

(Received April 20, 2017; accepted March 5, 2018)

Keywords: wrist rehabilitation robot, myoelectric sensor, mirror effect, interface system, clinical trials, electroencephalograph data, near-infrared spectroscopy

The authors have developed a practical wrist rehabilitation robot for hemiplegic patients. It consists of a desk equipped with mechanical rotating handle grips, sensors, a computer, and a display. Myoelectric sensors, which are bioelectric signal detectors, are used to monitor the extensor carpi radialis longus/brevis muscle and flexor carpi radialis muscle activity during training. Training effectiveness is ensured by monitoring the extension and flexion of the appropriate muscles. A feature of the system is that it operates as a dual-wrist system, developed to mirror the training between wrists, making autonomous training of either wrist possible. A user-friendly touch screen interface is provided; the screen displays progress data and images to encourage the patients towards completion of the necessary rehabilitation tasks critical to facilitate recovery. The developed robotic system is relatively compact and portable. The effectiveness of this system has been confirmed through clinical trials, and motivational effectiveness has been confirmed through electroencephalography (EEG)-based evaluation and direct survey.

\section{Introduction}

The effectiveness of repetitive rehabilitation training for stroke patients is clear from medical research. $^{(1-8)}$ Occupational therapists traditionally play key roles in rehabilitation training support through personal support for each and every patient as required. However, with the continued increase in demand for a limited number of therapists, the provision of assistive mechanisms to reduce the workload on such therapists is essential. While a number of assistive mechanisms are available, they tend to be physically large, heavy, complex to use, and lack the necessary feedback to encourage and inspire the patient to complete the necessary training critical for rehabilitation. ${ }^{(9-14)}$ To address these issues, the wrist training system outlined in this paper has been developed, tested in hospitals, and confirmed to be effective for rehabilitation.

*Corresponding author: e-mail: iyamamoto@nagasaki-u.ac.jp https://dx.doi.org/10.18494/SAM.2018.1901 


\section{Rehabilitation Robot and Its Interface}

A rehabilitation mechanism has been developed specifically to restore wrist movement particularly in the case of hemiplegia. The system schematic is shown in Fig. 1 and can be seen in use in Fig. 2. Myoelectric sensors are positioned on a patient's arms as shown in Fig. 3 to monitor the actuation of the extensor carpi radialis longus/brevis muscle and flexor carpi radialis muscles. The mechanism features two handles mounted on a desk as shown in Fig. 4. Each handle or grip is coupled to a servo motor to provide assisted actuation, position, and torque sensors to monitor user interaction. Finally, a computer screen is provided to customize system settings to the individual user's requirements and provide a display to show user progress and provide user motivation.

The usage of such myoelectric sensors (Fig. 3) has been found to be beneficial for monitoring rehabilitation training effectiveness in muscles. ${ }^{(15-18)}$ A major feature of this mechanism is the ability to mirror movement for training from the nonparetic hand, as expressed through interaction with the grip, to the paretic hand; this is referred to as mirror effect training. ${ }^{19)}$ This mirror effect has been found to be particularly effective for patients with advanced paralysis. If

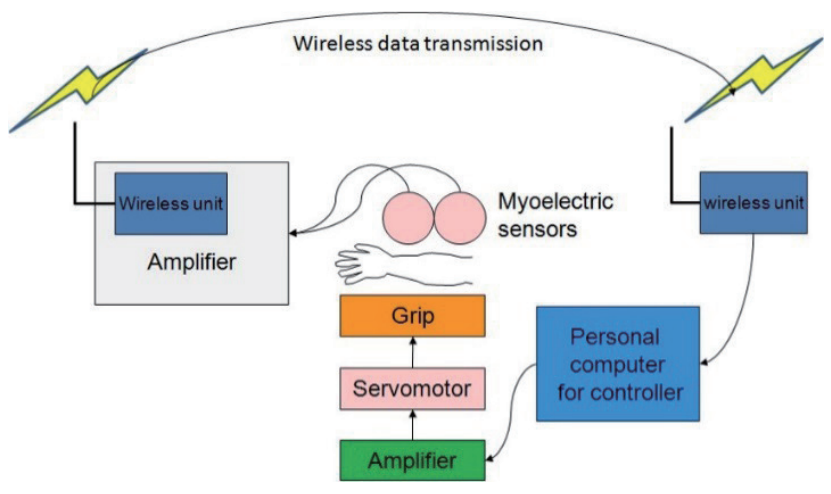

Fig. 1. (Color online) System schematic of wrist rehabilitation mechanism.

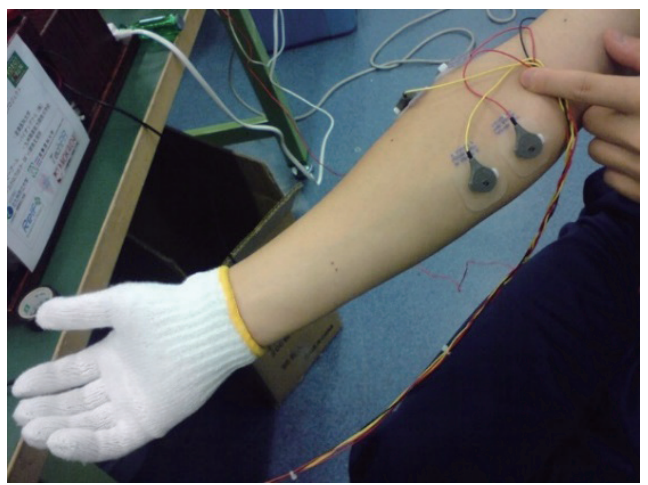

Fig. 3. (Color online) Positioning of myoelectric sensors.

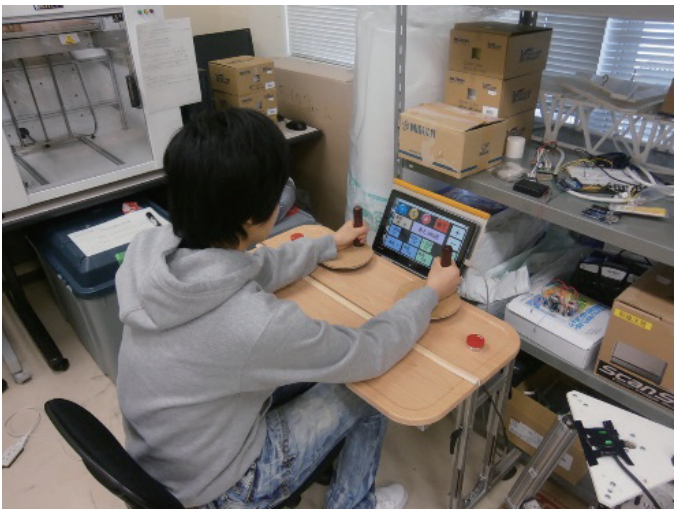

Fig. 2. (Color online) Wrist rehabilitation mechanism in operation.

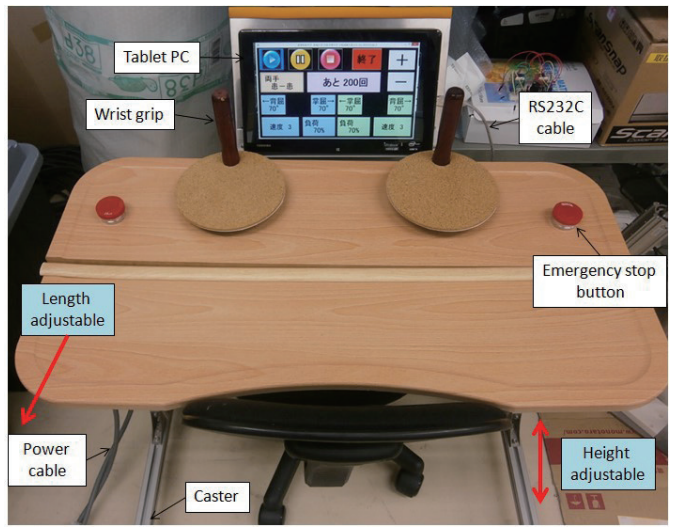

Fig. 4. (Color online) Wrist rehabilitation mechanism. 
a patient is unable to stably grip the handle, a strap is provided to ensure that the paretic hand maintains contact with the handle.

The developed wrist rehabilitation mechanism is relatively light and compact, making it suitable for both home and hospital use. The servo motors coupled to the grips are located under the desk panel, and customizable parameters are displayed on the user interface.

This wrist rehabilitation mechanism has been tested in five hospitals in Kyushu, Japan. On the basis of feedback from the tests, various changes have been made to make the system easier to use and more engaging.

Figure 5 shows the display that was initially developed. This screen provided excellent detail for rehabilitation specialists but was considered unfriendly for patients. Figure 6 shows a simplified display. The main parameters are the arm flexion angle shown in degrees, the torque expressed as a percentage of the maximum torque of $10 \mathrm{~N} \cdot \mathrm{m}(\sim 1 \mathrm{Kg} \cdot \mathrm{m})$, the number of repetitions (reps) to be completed, and the mode of operation; an emergency stop button is also provided on the desk itself.

The repetitive movement required for effective rehabilitation tends to become monotonous. ${ }^{(20)}$ To reduce this monotony, firstly, the remaining number of reps to be completed is displayed; in addition, a number of themes have been tested. Figure 6 shows a hamburger and a drink; this hamburger is put together from scratch, firstly, the buns, then the lettuce, etc. The completion is synchronized with the completion of the reps. The screen shown in Fig. 6 during clinical trials shows a slot machine screen. Other screens include creating sushi dishes and travel images to a wide variety of tourist destinations. Occasional sounds are also produced to assist attentiveness.

\section{Near-infrared Spectroscopy (NIRS) Measurement}

Formal measurement of the additional motivation provided by the improved screen was measured using NIRS-based neuroimaging. NIRS measures brain activation on the basis of concentration changes of oxygenated hemoglobin in the cerebral vessels. The neural activation of motor-related areas of a healthy subject was tested using NIRS (Fig. 7). Wrist movement rehabilitation tasks were performed using the original screen shown in Fig. 5, referred to as passive mode, and then tested using the newer display shown in Fig. 6, referred to as active mode. The experimental protocol consisted of three $30 \mathrm{~s}$ task reps with $40 \mathrm{~s}$ rests between the

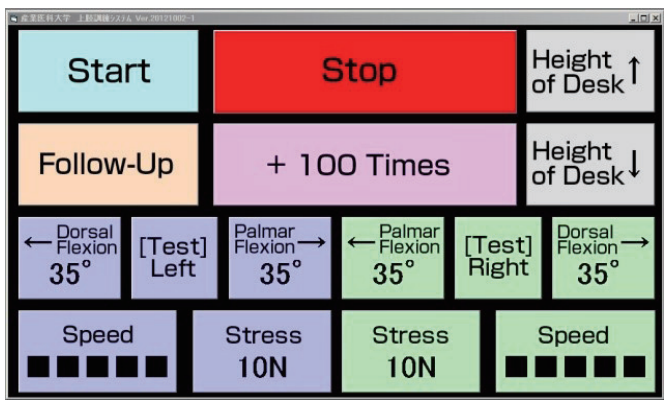

Fig. 5. (Color online) Original user interface.

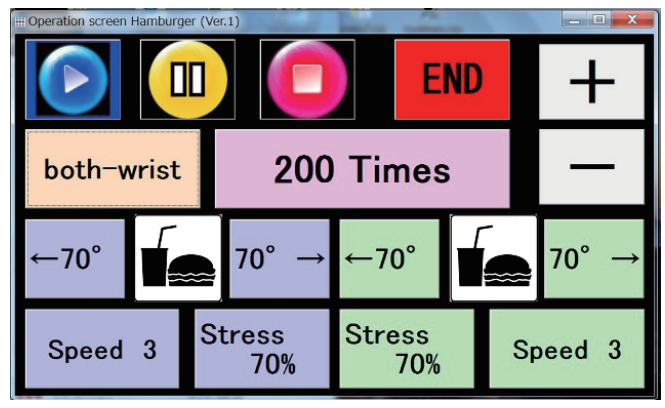

Fig. 6. (Color online) More user-friendly interface. 


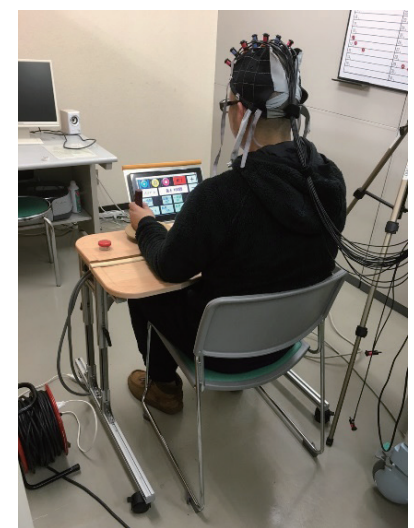

Fig. 7. (Color online) Neuroimaging trial.

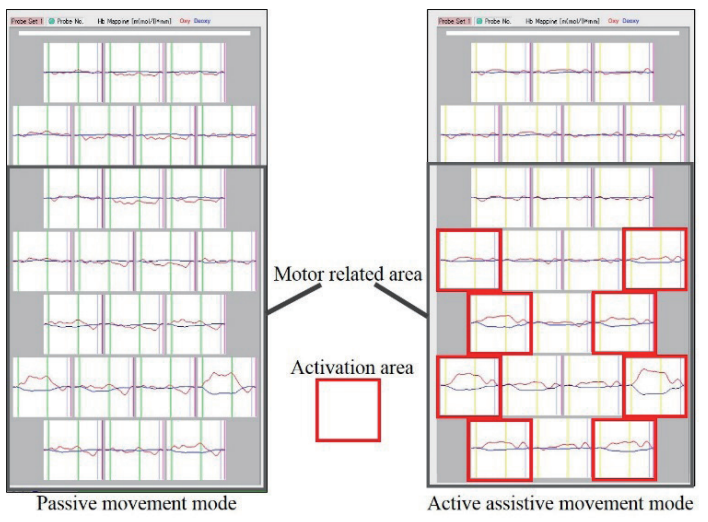

Fig. 8. (Color online) Test result of NIRS imaging.

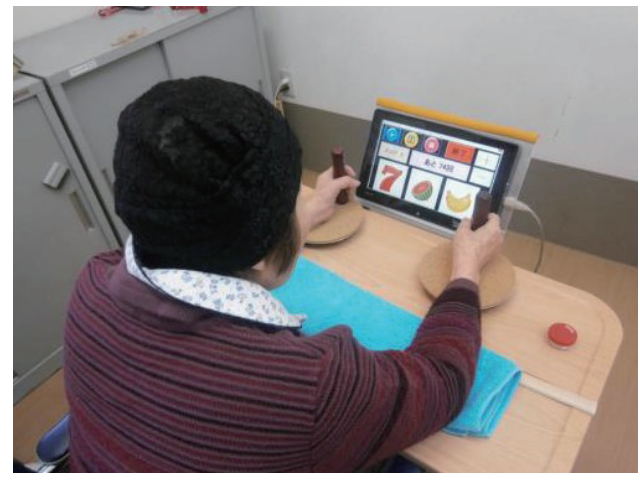

Fig. 9. (Color online) Clinical evaluation.

tasks. The results of the NIRS measurements are shown in Fig. 8. Each data block indicates the data obtained from a given cerebral location. The increased activation of motor-related areas during active assistance can be clearly seen. Areas that show significant variation between the passive and active modes have been outlined in red and labeled as "activation areas". On the basis of this NIRS data, the active assistance provided clearly indicates increased levels of motivation using the active screen.

\section{Effectiveness of Wrist Rehabilitation Robotic System Visual Interface}

A clinical trial was conducted to evaluate the effectiveness of the new visual interface for a stroke patient. An 81-year-old woman with left side hemiparesis resulting from cerebral infarction participated. The period of treatment is 5 months from the onset of the stroke. Both passive and active user interfaces were evaluated; in this case, the active theme was that of a slot machine (Fig. 9). At the end of each exercise, the participant was asked to assess the level of interest experienced during the exercise. The scale was a visual continuum (visual analogue scale, VAS) consisting of a $100 \mathrm{~mm}$ horizontal line with "Not interesting" on the left and "Very interesting" on the right. After using the passive interface, she gave a $46 \mathrm{~mm}$ VAS evaluation (gauged from the left). After using the active interface as shown in Fig. 9, she gave a 100 
mm VAS evaluation indicating "Very interesting". Therefore, the visual interface was highly evaluated by this particular patient.

The cost of the basic device is 500000 Japanese yen, and with the addition of all options, 1000000 Japanese yen. Mass production will reduce the cost. The use of this therapist that uses robotic technology is expected to be profitable.

\section{Conclusions}

A practical wrist rehabilitation robot has been developed, which provides dual-wrist mirror effect training for hemiplegic patients. A user interface system has been developed to provide motivation for the patient to complete the critical level of rehabilitation required to enable progression towards recovery. The wrist rehabilitation system has been clinically tested in hospitals, and increased user motivation has been proven through NIRS electroencephalography (EEG) data analysis as well as through direct survey data.

\section{Acknowledgments}

A part of this study was supported by a Grant-in-Aid for Scientific Research (B) in Japan, No. 25282169. The authors would like to express their sincere gratitude to related personnel for their research and development.

\section{References}

1 S. Saeki, Y. Matsushima, and K. Hachisuka: J. UOEH 30 (2008) 159.

2 G. B. Prange, M. J. A. Jannink, C. G. M. Groothuis-Oudshoorn, H. J. Hermens, and M. J. IJzerman: J. Rehabil. Res. Dev. 43 (2006) 171.

3 H. I. Krebs, N. Hogan, B. T. Volpe, M. L. Aisen, L. Edelstein, and C. Diels: Technol. Health Care 7 (1999) 419.

4 C. G. Burgur, P. S. Lum, P. C. Shor, and H. F. M. Van der Loos: J. Rehabil. Res. Dev. 37 (2000) 376.

5 S. Masiero, A. Celia, G. Rosati, and M. Armani: Arch. Phys. Med. Rehabil. 88 (2007) 142.

6 S. Hesse, G. Schulte-Tiggers, M. Konrad, M. A. Bardeleben, and C. Werner: Arch. Phys. Med. Rehabil. 84 (2003) 915.

7 S. Hesse, C. Werner, M. Pohl, S. Rueckriem, J. Mehrholz, and M. L. Lingnau: Stroke 36 (2005) 1960.

8 M. Ferraro, J. J. Palazzolo, J. Krol, H. I. Krebs, N. Hogan, and B. T. Volpe: Neurology 61 (2003) 1604.

9 G. D. Lee, W. W. Wang, K. W. Lee, S. Y. Lin, L. C. Fu, J. S. S. Lai, W. S. Chen, and J. J. J. Luh: Proc. ICCAS (2012) 1943-1948.

10 H. B. B. Wang, N. Du, H. Hou, J. S. Zhao, and H. N. Yu: Proc. ICAMechS (2012) 668-673.

11 X. Tu, J. Huang, L. Yu, Q. Xu, and J. He: Proc. IEEE RAS \& EMBS BioRob (2012) 1555-1560.

12 L. Shi, K. Jiang, Q. Wang, and Z. Liu: Proc. ICINIS (2012) 278-281.

13 Y. Kang and D. Jeon: Proc. SII (2012) 192-197.

14 C. Lv, L. Xie, W. Shao, H. L. Yu, Y. Wang, J. W. Wang, and N. Nan: Lect. Notes Comput. Sci. 7507 (2012) 96.

15 I. Yamamoto, N. Inagawa, K. Hachisuka, and Y. Nakanishi: Proc IEEE CME (2012) 557-560.

16 I. Yamamoto, N. Inagawa, K. Hachisuka, F. Wada, and A. Hachisuka: Proc. 2013 ICME (2013) 280-284.

17 I. Yamamoto, N. Inagawa, M. Matsui, K. Hachisuka, F. Wada, and A. Hachisuka: Bio-Med. Mater. Eng. 24 (2014) 123.

18 I. Yamamoto, M. Matsui, N. Inagawa, T. Tsuji, K. Hachisuka, F. Wada, and A. Hachisuka: Appl. Mech. Mater. 597 (2014) 397.

19 K. Hachisuka, F. Wada, M. Ochi, A. Hachisuka, S. Saeki, M. Nokatsuru, M. Hamada, I. Yamamoto, M. Matsui, and N. Inagawa: Proc. 9th World Stroke Congress (2014) 22-25.

20 Y. Nakanishi, F. Wada, S. Saeki, and K. Hachisuka: J. NeuroEng. Rehabil. (2014) 59. https://dx.doi. org/10.1186/1743-0003-11-59 


\section{About the Authors}

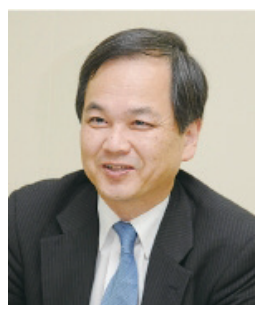

Ikuo Yamamoto received his Dr. Eng. degree in 1994 from Kyushu University, Japan and became a professor at Kyushu University in 2005, University of Kitakyushu in 2007, and Nagasaki University, Japan in 2013. He also became a GlobalScot member of Scotland, UK in 2017. His current research interests include robotics, mechatronics, and IoT.

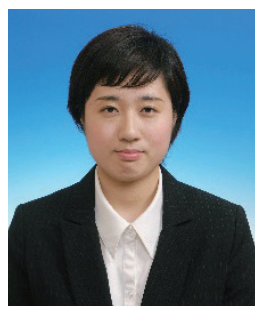

Miki Matsui received her B. Eng. Sc. degree from the University of Kitakyushu, Japan in 2014 and her M. Eng. Sc. degree from Nagasaki University, Japan in 2016. She works at Seiko Epson Corp., Japan. Her current research interests include robotics and mechatronics.

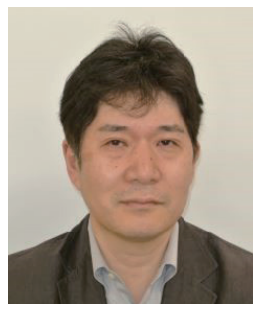

Toshio Higashi received his Ph.D. degree in science and engineering from Saga University, Japan in 2005. He now works as a professor at Nagasaki University Graduate School of Biomedical Sciences. His current research interests include neuroscience and neuro-rehabilitation.

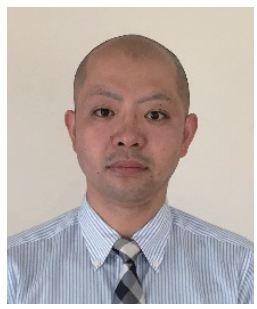

Naoki Iso received his Ph.D. degree from Nagasaki University Graduate School of Biomedical Sciences, Japan in 2016. He now works as an occupational therapist at Medical Corporation Tojinkai Miharadai Hospital and as a visiting researcher of Nagasaki University. His current research interests include the application of motor imagery to rehabilitation.

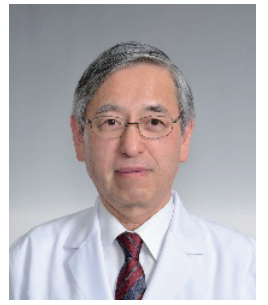

Kenji Hachisuka is a professor emeritus of the Department of Rehabilitation Medicine, University of Occupational and Environmental Health, Kitakyushu, Japan and has been the director of the Moji Medical Center, Japan Organization of Occupational Health and Safety since 2014. His clinical practice is in stroke, traumatic brain injury, and polio, and his research interests are in robot-assisted training and driving rehabilitation.

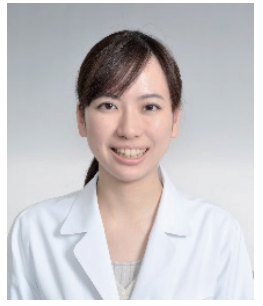

Akiko Hachisuka has been a visiting professor at the Department of Physical Medicine and Rehabilitation, Faculty of Medicine \& Dentistry, University of Alberta, Canada since 2016. She was an instructor on rehabilitation medicine at the University of Occupational and Environmental Health, Kitakyushu, Japan. Her clinical practice is in stroke and polio, and her research interests are in peripheral nerves, motor unit and control, and electrodiagnosis. 